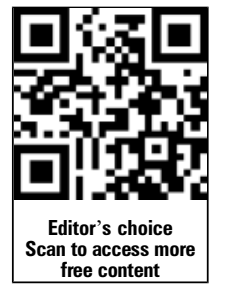

- Additional material is published online only. To view please visit the journal online (http://dx.doi.org/10.1136/ bjsports-2012-091905).

${ }^{1}$ Department of Orthopaedic Surgery, Arthroscopic Center Amager, Copenhagen University Hospital, Hvidovre, Denmark

${ }^{2}$ Department of Radiology, Rigshospitalet, Copenhagen University Hospital, Copenhagen, Denmark ${ }^{3}$ Aspetar Sports Groin Pain Center, Qatar Orthopedic and Sports Medicine Hospital, Doha, Qatar

\section{Correspondence to}

Dr Sonia Branci, Department of Radiology, Rigshospitalet, Copenhagen University Hospital, Blegdamsvej 9 Copenhagen OE 2100,

Denmark;

Sonia.Branci.01@regionh.dk

Received 21 October 2012 Accepted 27 November 2012 Published Online First 12 February 2013
To cite: Branci $S$, Thorborg K, Nielsen MB, et al. Br J Sports Med 2013;47:611-619.

\title{
Radiological findings in symphyseal and adductor-related groin pain in athletes: a critical review of the literature
}

\author{
Sonia Branci, ${ }^{1,2}$ Kristian Thorborg, ${ }^{1}$ Michael Bachmann Nielsen, ${ }^{2}$ Per Hölmich ${ }^{1,3}$
}

\begin{abstract}
Long-standing symphyseal and adductor-related groin pain is a common problem for many athletes, and requires a multidisciplinary approach. Radiological evaluation of symptomatic individuals is a cornerstone in the diagnostic workup, and should be based on precise and reliable diagnostic terms and imaging techniques. The authors performed a review of the existing original evidence-based radiological literature involving radiography, ultrasonography and MRI in athletes with long-standing symphyseal and adductor-related groin pain. Our search yielded 17 original articles, of which 12 were dedicated to MRI, four to radiography and one to ultrasonography. Four main radiological findings seem to consistently appear: degenerative changes at the pubic symphyseal joint, pathology at the adductor muscle insertions, pubic bone marrow oedema and the secondary cleft sign. However, the existing diagnostic terminology is confusing, and the interpretation of radiological findings would benefit from imaging studies using a more systematic approach.
\end{abstract}

\section{INTRODUCTION}

Long-standing groin pain is a common complaint for athletes involved in a number of sports such as soccer, rugby and ice hockey and causes significant morbidity and loss of sporting activity. ${ }^{1}$ It remains a complex clinical and radiological problem, as it affects a large anatomical region where many different pathological factors can contribute to the overall symptom pattern. ${ }^{2}$

The pubic symphysis and the adductor muscles are among the many anatomical structures potentially involved in causing athletic groin pain. ${ }^{3}$ Early studies ${ }^{4}$ describe clinical findings related to the adductor muscle group and the pubic symphysis, but do not include specific detailed radiological information on potential pathology. Over the past years, the adductor muscles, their insertion at the pubic bones and the pubic symphysis itself have been investigated with several radiological modalities, mainly conventional pelvic radiography, ultrasonography and MRI, whereas other modalities used extensively in the past (eg, isotope bone $\operatorname{scan}^{5}$ ) are nowadays obsolete due to more informative results obtained with ultrasonography and MRI. ${ }^{6}$

The aim of our review was to provide an overview of the existing literature assessing radiological findings in symphyseal and adductor-related longstanding groin pain in athletes with the radiological modalities radiography, ultrasonography and MRI, and thereby present an update on current radiological knowledge in this field. The term 'long-standing groin pain' is used in this review to describe non-specific groin pain in athletes with a duration of more than 6 weeks, non-specific meaning that this pain cannot objectively be related to the presence of fractures, hip and lumbar pathology, systemic diseases or neoplasms.

\section{METHODS}

Search

A search was made in MEDLINE for all relevant articles published until 1 June 2012 using the following combination of words ('adductor tendinosis' OR 'secondary cleft sign' OR 'adductor enthesis' OR 'pubic symphysis' OR 'adduction-related' OR 'adductor tendinitis' OR 'osteitis pubis' OR 'pubalgia' OR 'groin' OR 'adductor strain' OR 'tendon injury') AND ('validity' OR 'sensitivity' OR 'specific"' OR 'standards' OR 'false positive' OR 'false negative' OR 'reference' OR 'reliability' OR 'reproducibility' OR 'kappa' OR 'examination' OR 'examine' OR 'assess' OR ‘findings' OR 'results' OR 'register' OR 'interrater' OR 'inter rater' OR 'test') AND ('MR imaging' OR 'MRI' OR 'magnetic resonance' OR 'roentgen' OR 'radiography' OR 'X-ray' OR 'ultrasonography' OR 'ultrasound' OR 'sonography') AND ('sport' OR 'sports' OR 'football' OR 'soccer' OR 'athlete' OR 'athletic' OR 'athletes' OR 'sportsmen').

Abstracts of all articles listed on the search list were then read to include original studies dedicated to athletic long-standing groin pain emanating from the pubic symphysis and the adductor musculotendinous insertions. Articles were excluded if they were reviews, case reports or cadaver studies, if they were dealing with surgery, surgical results, sports hernias, hip or abdominal pathology or asymptomatic individuals and if they were not written in English or French. Articles were excluded if the main radiological modalities used were CT (as this review does not include traumatic injuries), isotope bone scan (rarely used nowadays) or herniography (used to diagnose hernias). However, if these modalities were merely additional examinations used to complement one of the main modalities of interest (radiography, ultrasonography or MRI), the study was still included. MRI studies where all scans were performed at a field strength below 1.0 Tesla were also excluded to ensure adequate quality of MRI. ${ }^{7}$

In this initial selection round, studies were included based on the information provided in their abstract. In the second round, included articles were read in full length and excluded if they 
were not reporting radiographic, ultrasonographic or MRI findings in athletes with long-standing groin pain emanating from the pubic symphysis and/or the adductor musculotendinous insertions, or if they were providing insufficient information about their radiological results. Lastly, reference lists of the retrieved papers were hand-searched to identify further relevant studies.

\section{Search results}

The MEDLINE search yielded 252 results. Of these, 236 were excluded, leaving 17 articles for inclusion in this review: 4 articles involve radiography as their main radiological modality, 1 involves ultrasound (US) and 12 involve MRI (see online supplementary figure S1). Most of the retrieved studies are based on a combination of clinical, radiological and sometimes surgical data. In the following sections, we extract all relevant radiological results from these studies, which implies less emphasis on clinical and surgical results.

Conventional radiography is the original modality used to assess athletes with symphyseal and adductor-related groin pain. It is still prevalent today as it depicts pelvic bony and articular structures. Patients can be examined in a supine position or standing upright (weight-bearing) to show the presence of symphyseal instability. ${ }^{8}$ Ultrasonography provides dynamic real-time images and is particularly adapted for examining superficial soft tissues (tendons and muscles) in the groin area. MRI is useful in imaging parts of the body with little density contrast (such as soft tissues), and provides images with high resolution and contrast by using strong magnetic fields and non-ionising radiation, combined with a large field-of-view.

Retrieved studies were evaluated for our analysis according to the following parameters: study design and participants, presence of control groups, inclusion and exclusion criteria, clinical findings, radiological protocols and evaluation and reliability of radiological findings (tables $1-4)$.

Ideally, study groups should be as homogeneous as possible in terms of age, sex and sports practiced to ensure that participants are comparable: the frequency of degenerative musculoskeletal changes increases with age, ${ }^{9}$ there are anatomical variations in the pelvic region between sexes, and different sport types affect pelvic musculotendinous structures differently depending on the predominant movement patterns. Inclusion and exclusion criteria should be as well defined as possible to ensure that study participants are selected properly and are representative of the pathology of interest. Symptomatic cases should be compared with well-matched asymptomatic controls to evaluate differences between groups. Clinical information should be available in detail to document the diagnostic steps suggesting potential pathology. Radiological protocols should be identical for all study participants to ensure comparable data, images should be evaluated by blinded examiners according to a predefined detailed checklist and the reproducibility of radiological findings should be assessed to determine the quality of this checklist.

\section{RESULTS}

\section{Study participants}

In one study, ${ }^{10}$ individuals were evaluated radiologically with three sequential MRI scans over 4 months. In all other studies, participants were examined radiologically once without further follow-up, and the radiological results are therefore crosssectional. In the following, the term 'case-study group' refers to the study group of interest regardless of whether there is a control group or not.
In six MRI studies, all participants were men. ${ }^{10-15}$ In five MRI studies, ${ }^{16-20}$ two radiographic studies ${ }^{21} 22$ and the US study, ${ }^{23}$ the study population comprised male and female individuals, whereas in one MRI study ${ }^{24}$ and one radiographic study, ${ }^{25}$ sex was not explicitly cited. They were generally young, and the age range covered in MRI studies was $17-40$ years, in radiographic studies 13-61 years and in the US study 14-57 years. Inclusion criteria for the case-study groups were provided in all included studies, but were explained in detail in only three MRI articles. ${ }^{10} \quad 1124$ Nine studies provided no information about exclusion criteria. ${ }^{10} \quad 12-14 \quad 18 \quad 21-23 \quad 25$ In 14 of 17 studies, ${ }^{10-19} 212425$ case-study groups were composed entirely of athletes.

\section{Control groups}

Radiological findings in a symptomatic group are best evaluated if they are compared with those of an asymptomatic matched control group, as differences between groups could point at possible aetiological symptom-provoking factors. In 10 of 17 studies, a control group was included. There was a total of six MRI case-control studies, ${ }^{12} 1416171920$ of which one had two control groups $^{12}$ and five had one control group. ${ }^{14} 16171920$ Three radiographic studies had one $\mathrm{e}^{21}$ or $\mathrm{two}^{26}$ control groups. In the US study, ${ }^{23}$ patients in the case-study group functioned as their own controls, as their symptomatic and asymptomatic sides were compared with each other. Control groups consisted of athletes in four studies, ${ }^{14} 161719$ of sedentary individuals in one MRI study ${ }^{20}$ and two radiographic studies ${ }^{21} 26$ and of both athletes and sedentary individuals in one $\mathrm{MRI}^{12}$ and one radiographic study. ${ }^{25}$

\section{Clinical findings}

A correct documentation of clinical findings is essential when interpreting radiological results subsequently. Both should ideally be held up against each other to avoid misinterpretation of radiological signs that may be incidental findings unrelated to the actual symptoms experienced by the patients.

Clinical examination of study participants varies among retrieved studies. Overall, 14 of 17 studies evaluated participants clinically. Two radiographic studies, ${ }^{21} 22$ the US study ${ }^{23}$ and 11 of 12 MRI studies ${ }^{10-13} \quad 15-2024$ included a preliminary clinical examination of all case-study participants, whereas 1 $\mathrm{MRI}^{14}$ and 2 radiographic studies ${ }^{25} 26$ mentioned none. In two MRI studies, ${ }^{11} 24$ all study participants underwent a reproducible clinical examination focused on the groin and lower abdominal areas. $^{27}$

\section{Radiological protocols}

To ensure homogeneity of research results, radiological evaluation of all study participants should ideally be performed with the same radiological equipment and according to a predefined identical protocol. In 10 of 17 studies, radiological examinations were identical for all participants. ${ }^{10} 11$ 13-17 192426

In one radiographic study, ${ }^{26}$ pelvic radiographs were obtained in a supine position for all participants, whereas in another study, ${ }^{25}$ pelvic films were recorded differently for cases and controls. In two radiographic studies, ${ }^{21} 22$ the position in which pelvic films were taken was not described. Individuals participating in the US study ${ }^{23}$ were examined on the same machine and at the same transducer frequency. Neither the radiographic nor the ultrasonographic protocols were reproducible.

Among the retrieved MRI studies, radiological protocols included at least one MRI scan per participant per study. In three studies, patients had undergone additional radiographs of 
Table 1 MRI case studies

\begin{tabular}{|c|c|c|c|c|c|c|}
\hline Study & Slavotinek et al (2005) & Schilders et al (2007) & Schilders et al (2009) & Kunduraciogluet al (2007) & Albers et al (2001) & Lovell et al (2006) \\
\hline $\begin{array}{l}\text { Case-study group } \\
\text { participants (incl. } \\
\text { sports type, gender, } \\
\text { age) }\end{array}$ & $\begin{array}{l}52 \text { athletes (Australian football) } \\
\text { Male. Age: } 17-31\end{array}$ & $\begin{array}{l}24 \text { athletes (19 soccer, } 2 \text { rugby, } \\
2 \text { runners, } 1 \text { squash) } \\
\text { Male. Age: } 19-41\end{array}$ & $\begin{array}{l}28 \text { recreational athletes (14 soccer, } \\
6 \text { rugby, } 3 \text { golf, } 2 \text { squash, } 2 \text { cycling, } 1 \\
\text { swimming, } 1 \text { triathlon) } \\
\text { Gender unknown. Age } 18-50\end{array}$ & $\begin{array}{l}22 \text { athletes ( } 21 \text { soccer, } 1 \text { handball) } \\
\text { Male. Age } 17-43\end{array}$ & $\begin{array}{l}30 \text { athletes ( } 16 \text { soccer, } 5 \text { football, } \\
\text { other sports) } \\
29 \text { Male, } 1 \text { Female. Age } 18-40\end{array}$ & $\begin{array}{l}19 \text { athletes (Australian } \\
\text { football) } \\
\text { Male. Age 15-17 }\end{array}$ \\
\hline Inclusion criteria & $\begin{array}{l}\text { Athletes from two teams of the } \\
\text { Australian Football League with } \\
\text { or without groin pain }\end{array}$ & $\begin{array}{l}\text { Competitive athletes, clinical } \\
\text { adductor dysfunction, failure of } \\
\text { non-operative treatment (rest, ice, } \\
\text { NSAID, physiotherapy) }\end{array}$ & $\begin{array}{l}\text { Recreational athletes, participate in } \\
\text { sports }<4 \text { days/week, clinical adductor } \\
\text { dysfunction, failure of non-operative } \\
\text { treatment (rest, ice, NSAID, } \\
\text { physiotherapy) }\end{array}$ & $\begin{array}{l}\text { Elite athletes referred to sports clinic with } \\
\text { a possible diagnosis of osteitis pubis and } \\
\text { duration of symptoms }>6 \text { weeks }\end{array}$ & $\begin{array}{l}\text { Patients with pubalgia who are } \\
\text { surgically confirmed as having } \\
\text { pubalgia caused by abnormal } \\
\text { musculofascial abnormalities } \\
\text { (PAMA) }\end{array}$ & $\begin{array}{l}\text { Athletes from the Australian } \\
\text { Institute of Sports, training } 2 \\
\text { months prior to start of the } \\
\text { study }\end{array}$ \\
\hline Exclusion criteria & $\begin{array}{l}\text { Athletes with pain not located } \\
\text { in the pubic symphysis, pubic } \\
\text { bones, adductor area or lower } \\
\text { abdominal area }\end{array}$ & $\begin{array}{l}\text { Any clinical evidence of sports hernia } \\
\text { or osteitis pubis. Any clinical or } \\
\text { radiographic evidence of pathological } \\
\text { involvement of hip joints }\end{array}$ & $\begin{array}{l}\text { Any clinical evidence of sports hernia or } \\
\text { osteitis pubis. Any clinical or } \\
\text { radiographic evidence of pathological } \\
\text { involvement of hip joints }\end{array}$ & Not described & Not described & Not described \\
\hline $\begin{array}{l}\text { Clinical findings } \\
\text { (1) type of } \\
\text { examination } \\
\text { (2)findings }\end{array}$ & $\begin{array}{l}\text { (1)Not reproducible } \\
\text { (2) } 23 \text { athletes have current } \\
\text { groin pain, } 18 \text { a history of groin } \\
\text { pain; } 39 \text { have tenderness on } \\
\text { palpation of symphysis or } \\
\text { adductor origin }\end{array}$ & $\begin{array}{l}\text { (1)Reproducible (a.m. Hölmich) } \\
\text { (2) all athletes have unilateral } \\
\text { adductor dysfunction at clinical } \\
\text { examination }\end{array}$ & $\begin{array}{l}\text { (1)Reproducible (a.m. Hölmich) } \\
\text { (2) all athletes have unilateral adductor } \\
\text { dysfunction at clinical examination }\end{array}$ & $\begin{array}{l}\text { (1) Not described } \\
\text { (2) all athletes have tenderness on } \\
\text { palpation of symphysis. } 6 \text { have bilateral } \\
\text { and } 16 \text { have unilateral groin pain. } 6 \\
\text { athletes have pain at adductor muscle } \\
\text { origin }\end{array}$ & $\begin{array}{l}\text { (1) Not described } \\
\text { (2) } 17 \text { athletes with bilateral and } 13 \\
\text { athletes with unilateral groin pain }\end{array}$ & $\begin{array}{l}\text { (1) Not described } \\
\text { (2) one athlete with groin } \\
\text { pain and tenderness at pubic } \\
\text { symphysis at the start of the } \\
\text { study }\end{array}$ \\
\hline $\begin{array}{l}\text { Diagnostic entity used } \\
\text { by authors }\end{array}$ & Not defined & Clinical adductor dysfunction & Clinical adductor dysfunction & Osteitis pubis & Pubalgia & Osteitis pubis \\
\hline $\begin{array}{l}\text { Radiology protocol } \\
\text { (1) field strength and } \\
\text { (2) sequences }\end{array}$ & $\begin{array}{l}\text { (1) } 1.5 \mathrm{~T} \text { and } 1.0 \mathrm{~T} \text { and }(2) \\
\text { coronal and axial T1\& T2 FatSat }\end{array}$ & $\begin{array}{l}\text { (1) } 1.5 \mathrm{~T} \text { and (2) coronal STIR, axial } \\
\text { oblique T1 and axial oblique and } \\
\text { sagittal T1 FatSat postintravenous } \\
\text { gadolinium }\end{array}$ & $\begin{array}{l}\text { (1) } 1.5 \mathrm{~T} \text { and (2) coronal STIR, axial T2, } \\
\text { axial oblique T2 FatSat and axial } \\
\text { oblique and sagittal T1 FatSat } \\
\text { postintravenous gadolinium }\end{array}$ & $\begin{array}{l}\text { (1) } 1.5 \mathrm{~T} \text { and (2) coronal T1 and STIR, } \\
\text { axial T2 }\end{array}$ & $\begin{array}{l}\text { (1) } 1.5 \mathrm{~T} \text { and (2) coronal and axial } \\
\text { T1\&T2, axial STIR }\end{array}$ & $\begin{array}{l}\text { (1) } 1.5 \mathrm{~T} \text { and (2) coronal } \\
\text { T1\&STIR, axial STIR, athletes } \\
\text { scanned three times (at start } \\
\text { and after } 2 \text { and } 4 \text { months) }\end{array}$ \\
\hline $\begin{array}{l}\text { Additional radiological } \\
\text { examinations }\end{array}$ & None & Pelvic radiographs & Pelvic radiographs & None & None & $\begin{array}{l}\text { Extra MRI scan if athlete } \\
\text { develops pain during study }\end{array}$ \\
\hline $\begin{array}{l}\text { Evaluation of MRI } \\
\text { scans }\end{array}$ & $\begin{array}{l}2 \text { Radiologists blinded to } \\
\text { clinical details. Diagnosis by } \\
\text { consensus. } \\
\text { BMO (graded 0-3 and extent } \\
>2 \mathrm{~cm} \text { ) } \\
\text { Degenerative changes at } \\
\text { symphyseal joint }\end{array}$ & $\begin{array}{l}1 \text { Radiologist with full clinical details } \\
\text { Presence of contrast enhancement at } \\
\text { adductor muscle origin }\end{array}$ & $\begin{array}{l}1 \text { Radiologist with full clinical details } \\
\text { Presence of contrast enhancement at } \\
\text { adductor muscle origin }\end{array}$ & $\begin{array}{l}1 \text { Radiologist with full clinical details } \\
\text { BMO (not graded) } \\
\text { Degenerative changes at symphyseal joint }\end{array}$ & $\begin{array}{l}2 \text { Radiologists blinded to clinical } \\
\text { details. Diagnosis by consensus } \\
\text { BMO (not graded) } \\
\text { Degenerative changes at } \\
\text { symphyseal joint } \\
\text { Musculotendinous structures of the } \\
\text { groin and abdominal wall. Inguinal } \\
\text { hernia, hip and SI joints }\end{array}$ & $\begin{array}{l}2 \text { Radiologists blinded to } \\
\text { clinical details. Diagnosis by } \\
\text { consensus } \\
\text { BMO (graded 0-3) } \\
\text { Degenerative changes at } \\
\text { symphyseal joint } \\
\text { Adductor muscle origin } \\
\text { Inguinal hernia }\end{array}$ \\
\hline $\begin{array}{l}\text { Reproducibility of } \\
\text { radiology }\end{array}$ & Interobserver agreement & None & None & None & None & None \\
\hline 'Gold standard' used & None & $\begin{array}{l}\text { Ultrasound-guided injection of local } \\
\text { anaesthetic and steroid in } \\
\text { symphyseal joint to treat pain }\end{array}$ & $\begin{array}{l}\text { Ultrasound-guided injection of local } \\
\text { anaesthetic and steroid in symphyseal } \\
\text { joint to treat pain }\end{array}$ & None & $\begin{array}{l}\text { Surgery (modified Bassini } \\
\text { hernioplasty) }\end{array}$ & None \\
\hline Conclusions/results & $\begin{array}{l}17 \text { of } 39 \text { athletes have groin } \\
\text { tenderness, and } 19 \text { of the total } \\
52 \text { athletes have severe BMO } \\
\text { Interobserver agreement for } \\
\text { grading of BMO : } \kappa=0.85 \\
\text { (good) }\end{array}$ & $\begin{array}{l}17 \text { athletes with contrast } \\
\text { enhancement at adductor muscle } \\
\text { origin (enthesitis): } 12 \text { athletes with } \\
\text { gradual onset of pain, } 5 \text { with acute } \\
\text { onset } \\
7 \text { athletes without contrast } \\
\text { enhancement at adductor muscle } \\
\text { origin } \\
\text { No evidence of femoroacetabular } \\
\text { impingement on radiographs }\end{array}$ & $\begin{array}{l}13 \text { athletes with contrast enhancement } \\
\text { at adductor muscle origin (enthesitis) } \\
15 \text { athletes without contrast } \\
\text { enhancement at adductor muscle origin } \\
\text { No evidence of femoroacetabular } \\
\text { impingement on radiographs }\end{array}$ & $\begin{array}{l}14 \text { athletes with BMO } \\
\text { Degenerative changes present in } 50 \% \\
\text { of the group of athletes }\end{array}$ & $\begin{array}{l}21 \text { athletes with BMO, which in } 20 \\
\text { corresponds to side of surgery } \\
18 \text { athletes with increased signal at } \\
\text { adductor muscle origin } \\
6 \text { athletes with increased signal in } \\
\text { pectineus muscle, } 27 \text { with } \\
\text { attenuation of abdominal } \\
\text { musculofascial layers }\end{array}$ & $\begin{array}{l}1 \text { athlete with groin pain at } \\
\text { start of study. } 4 \text { Athletes } \\
\text { develop pain during study } \\
\text { Initially BMO in } 11 \text { athletes. } \\
\text { Increase in severity of BMO } \\
\text { during the training season. } \\
\text { BMO present in } 2 / 3 \text { of } \\
\text { asymptomatic athletes }\end{array}$ \\
\hline
\end{tabular}

BMO, bone marrow oedema. 
Table 2 MRI case-control studies

\begin{tabular}{|c|c|c|c|c|c|c|}
\hline Study & Cunningham et al(2007) & Robinson et al(2004) & Zoga et al(2008) & Brennan et al(2005) & Verrall et al(2001) & Paajanen et al(2008) \\
\hline $\begin{array}{l}\text { Case-study group } \\
\text { participants (incl. } \\
\text { sports type, gender, } \\
\text { age) }\end{array}$ & $\begin{array}{l}100 \text { athletes (soccer) } \\
95 \text { Male, } 5 \text { Female. } \\
\text { Age: } 17-38\end{array}$ & $\begin{array}{l}52 \text { athletes ( } 35 \text { soccer, } \\
13 \text { rugby, } 4 \text { athletics), of } \\
\text { whom } 27 \text { previous surgery } \\
51 \text { Male, } 1 \text { Female. Age 17-36 }\end{array}$ & $\begin{array}{l}141 \text { patients (127 athletes: } \\
41 \text { American football, } \\
23 \text { soccer, } 15 \text { running, } \\
14 \text { hockey, } 34 \text { others) } \\
134 \text { Male, } 7 \text { Female. } \\
\text { Age } 17-71\end{array}$ & $\begin{array}{l}18 \text { athletes ( } 15 \text { soccer, } 3 \text { rugby) } \\
\text { Male. Age } 19-32\end{array}$ & $\begin{array}{l}89 \text { athletes (Australian } \\
\text { football) } \\
\text { Male. Age 17-33 }\end{array}$ & $\begin{array}{l}16 \text { athletes (13 soccer, } \\
1 \text { cross-country skier, } 1 \text { runner, } \\
1 \text { ice-hockey) } \\
14 \text { Male, } 2 \text { Female. Age } 22-38\end{array}$ \\
\hline Controls & $\begin{array}{l}100 \text { athletes (50 rowers, } \\
50 \text { soccer) } \\
\text { Sex unknown. Age :18-28 }\end{array}$ & $\begin{array}{l}6 \text { athletes ( } 5 \text { soccer, } 1 \text { rugby) } \\
\text { Male. Age } 17-37\end{array}$ & $\begin{array}{l}25 \text { (physical activity unknown) } \\
\text { Male. Age 18-39 }\end{array}$ & $\begin{array}{l}70 \text { athletes (rowers) } \\
\text { Male, Age 17-34 }\end{array}$ & $\begin{array}{l}\text { Group 1: } 17 \text { distance runners } \\
\text { Group 2: } 10 \text { sedentary men }\end{array}$ & $\begin{array}{l}20 \text { athletes (10 soccer, } 10 \text { ice-hockey) } \\
\text { Male. Age } 19-27\end{array}$ \\
\hline Inclusion criteria & $\begin{array}{l}\text { Cases: Debilitating groin } \\
\text { pain and symptoms and } \\
\text { signs at pubic symphysis } \\
\text { Controls: Asymptomatic, } \\
\text { hip pain and pain from } \\
\text { sacroiliac joints }\end{array}$ & $\begin{array}{l}\text { Cases: Groin pain }>3 \text { months } \\
\text { Controls: no groin symptoms } \\
\text { or injury, no groin surgery }\end{array}$ & $\begin{array}{l}\text { Cases: Patients referred with } \\
\text { diagnoses 'athletic pubalgia" } \\
\text { and 'sports hernia" } \\
\text { Controls: Asymptomatic }\end{array}$ & $\begin{array}{l}\text { Cases: Patients referred with suspicion } \\
\text { of groin injury } \\
\text { Controls: Asymptomatic }\end{array}$ & $\begin{array}{l}\text { Cases: recruited at end of } \\
\text { pre-season after } 6 \text { weeks } \\
\text { intensive training } \\
\text { Control 1: age-matched, min. } \\
6 \text { weeks intensive training } \\
\text { Control 2: no prior history of } \\
\text { groin pain, no physical } \\
\text { exercise within } 6 \text { weeks, } \\
\text { age-matched }\end{array}$ & $\begin{array}{l}\text { Cases: Osteitis pubis (established by } \\
\text { exclusion of other groin disorders, typical } \\
\text { clinical history and signs, pelvic } \\
\text { radiographs isotope bone scan and MRI) } \\
\text { Controls: Asymptomatic }\end{array}$ \\
\hline Exclusion criteria & $\begin{array}{l}\text { Cases: Sports hernia at } \\
\text { clinical examination } \\
\text { before referral } \\
\text { Controls: groin pain }\end{array}$ & $\begin{array}{l}\text { Cases: Acute groin injury, } \\
\text { insufficient clinical and } \\
\text { surgical details available }\end{array}$ & $\begin{array}{l}\text { Cases: Inadequate MRI of } \\
\text { pubic region. Images older } \\
\text { than } 120 \text { days from time of } \\
\text { physical examination }\end{array}$ & Not described & Not described & $\begin{array}{l}\text { Cases: Inguinal hernias, iliopsoas and } \\
\text { abdominal muscle-related pain. chronic } \\
\text { prostatitis.tendinitis of the groin, bursitis } \\
\text { or hip disorders }\end{array}$ \\
\hline $\begin{array}{l}\text { Clinical findings } \\
\text { (1) type of } \\
\text { examination } \\
\text { (2) findings }\end{array}$ & $\begin{array}{l}\text { (1) Not described } \\
\text { (2) Groin pain for average } \\
\text { of } 3 \text { months }\end{array}$ & (1 and 2) Not described & $\begin{array}{l}\text { (1) Not reproducible but } \\
\text { details given } \\
\text { (2) } 93 \text { positive for rectus } \\
\text { abdominis tendon lesion, } 15 \\
\text { for adductor compartment } \\
\text { lesion,71 for both, } 16 \text { for } \\
\text { osteitis pubis (not defined) }\end{array}$ & None & $\begin{array}{l}\text { (1) Not reproducible but } \\
\text { details given } \\
\text { (2) } 52 \text { athletes positive for } \\
\text { current groin symptoms and } \\
\text { signs (tenderness on } \\
\text { palpation of symphysis and } \\
\text { superior pubic rami) }\end{array}$ & $\begin{array}{l}\text { (1) Not described } \\
\text { (2) All cases have tenderness of pubic } \\
\text { symphysis }>3 \text { months. } 3 \text { cases with } \\
\text { adductor-type pain on palpation }\end{array}$ \\
\hline $\begin{array}{l}\text { Diagnostic entity } \\
\text { used by authors }\end{array}$ & Osteitis pubis & Adductor dysfunction & Athletic pubalgia & Osteitis pubis & Osteitis pubis & Osteitis pubis \\
\hline $\begin{array}{l}\text { Radiology protocol } \\
\text { (1) Field strength } \\
\text { (2) Sequences }\end{array}$ & $\begin{array}{l}\text { (1) } 1.5 \mathrm{~T} \\
\text { (2)Coronal T1\& STIR, } \\
\text { axial T2 }\end{array}$ & $\begin{array}{l}\text { (1) } 1.5 T \\
\text { (2) Coronal: T1\& STIR, axial } \\
\text { T2. Oblique axial: T1, T2 } \\
\text { FatSat, T1Fatsat } \\
\text { postgadolinium }\end{array}$ & $\begin{array}{l}\text { (1) Cases: various (117 at } \\
1.5 \mathrm{~T}, 2 \text { at } 3.0 \mathrm{~T}, 16 \text { at } 0.2- \\
0.3 \mathrm{~T}, 6 \text { at } 0.6-0.7 \mathrm{~T}) \\
\text { Controls: } 1.5 \mathrm{~T} \\
\text { (2) Protocols not defined }\end{array}$ & $\begin{array}{l}\text { (1) } 1.5 T \\
\text { (2) Cases: coronal T1 and STIR, axial T2 } \\
\text { Controls: coronal STIR only }\end{array}$ & $\begin{array}{l}\text { (1) } 1.5 \text { and } 1.0 \mathrm{~T} \\
\text { (2) Coronal and axial:T1 and } \\
\text { T2 FatSat }\end{array}$ & $\begin{array}{l}\text { (1) 1,0 T } \\
\text { (2) Coronal and axial: T1 and STIR }\end{array}$ \\
\hline $\begin{array}{l}\text { Additional radiologic } \\
\text { examinations }\end{array}$ & None & None & None & $\begin{array}{l}\text { Pelvic radiographs } \\
\text { Fluoroscopy-guided injection of contrast } \\
\text { in symphyseal joint }\end{array}$ & None & Pelvic radiographs and isotope bone scan \\
\hline $\begin{array}{l}\text { Evaluation of MRI } \\
\text { scan }\end{array}$ & $\begin{array}{l}2 \text { Radiologists blinded to } \\
\text { side of symptoms. } \\
\text { Diagnosis by consensus. } \\
\text { Presence of secondary } \\
\text { cleft sign } \\
\text { Degenerative changes at } \\
\text { symphyseal joint }\end{array}$ & $\begin{array}{l}2 \text { Radiologists blinded to } \\
\text { clinical details. Diagnosis by } \\
\text { consensus } \\
\text { BMO (graded 0-2) } \\
\text { Degenerative changes at } \\
\text { symphyseal joint } \\
\text { Musculotendinous structures of } \\
\text { the groin \& abdominal wall } \\
\text { Graded at } 1 \text { and } 2 \text { reading } \\
(0-2)\end{array}$ & $\begin{array}{l}3 \text { Radiologists blinded to } \\
\text { clinical details. Diagnosis by } \\
\text { consensus } \\
\text { BMO (not graded) } \\
\text { Osteitis pubis (BMO with } \\
\text { degenerative changes at } \\
\text { symphyseal joint) } \\
\text { Abnormal rectus abdominis } \\
\text { and adductor tendons } \\
\text { Presence of secondary cleft } \\
\text { sign }\end{array}$ & $\begin{array}{l}2 \text { Radiologist: } 1 \text { blinded and } \\
1 \text { performing fluoroscopy-guided } \\
\text { injections.Diagnosis by consensus } \\
\text { Presence of secondary cleft sign } \\
\text { BMO (not graded) } \\
\text { Degenerative changes at symphyseal } \\
\text { joint }\end{array}$ & $\begin{array}{l}2 \text { Radiologists blinded to } \\
\text { clinical details. Diagnosis by } \\
\text { consensus } \\
\text { BMO (graded 0-3 and } \\
\text { extent }>2 \mathrm{~cm} \text { ) } \\
\text { Degenerative changes at } \\
\text { symphyseal joint } \\
\text { Abnormal conjoint tendon } \\
\text { Inguinal hernia }\end{array}$ & $\begin{array}{l}2 \text { Radiologists blinded to clinical details. } \\
\text { Diagnosis by consensus } \\
\text { BMO (graded } 0-3 \text { ) } \\
\text { Degenerative changes at symphyseal joint } \\
\text { Abnormal conjoint tendon } \\
\text { Abnormal adductor enthesis }\end{array}$ \\
\hline
\end{tabular}




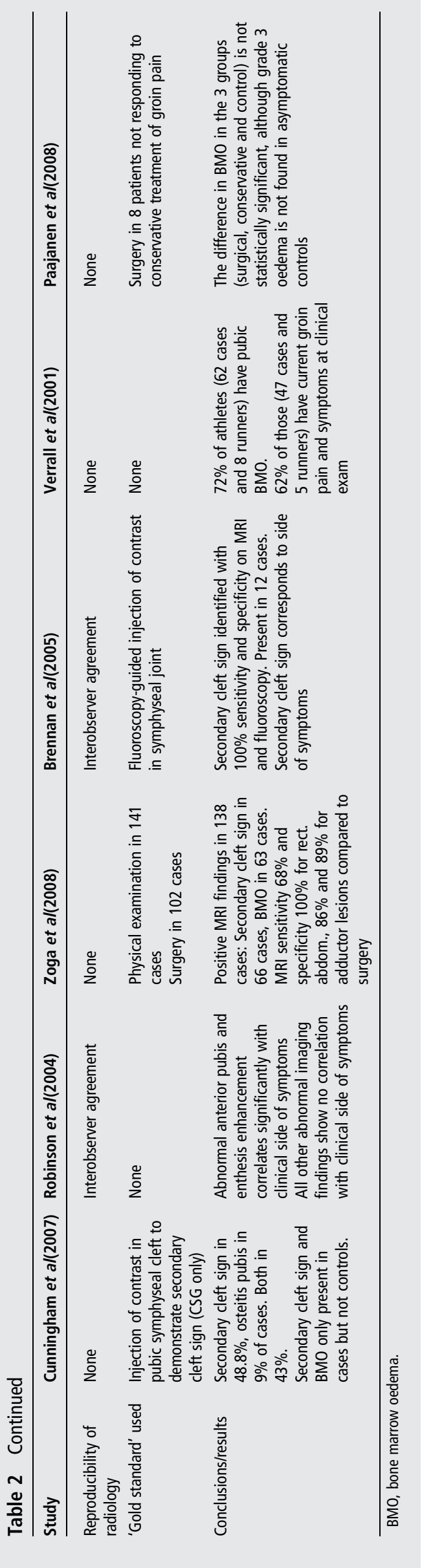

the pelvic region, ${ }^{11} 1419$ isotope bone scans ${ }^{19}$ and MR arthrograms. ${ }^{11}$ In all but three studies, ${ }^{12} 1520$ all MRI scans were performed on the same machine for all participants. The MRI scanning protocols were cited in all articles, but were not equally detailed. They were detailed enough to be reproducible in nine articles, ${ }^{1011} 13-171924$ but were less specific in two articles. ${ }^{12}{ }^{18}$ In one study, ${ }^{20}$ MRI scans had been performed in many different institutions with numerous imaging systems and variable protocols.

\section{Reliability of radiology}

Intraobserver and interobserver agreements are important factors in measuring the reliability of radiological results. Ideally, images should be interpreted similarly by different radiologists, regardless of the personal level of experience and geography.

A total of 4 out of 17 articles (1 radiographic ${ }^{26}$ and 3 MRI studies $\left.{ }^{14-16}\right)$ included an assessment of the interobserver variation between two radiologists, but none included one of the intraobserver variation. Blinding of radiologists varied considerably in all MRI studies. In 8 of 17 studies, radiological evaluation was conducted independently by several radiologists blinded to all clinical information concerning study participants, and radiological diagnosis was obtained by consensus.

\section{DISCUSSION}

The aim of our review is to provide an overview of the existing scientific evidence on radiological pathological findings in athletes with long-standing symphyseal and adductor-related groin pain. Our literature search yielded only 17 original articles dedicated to this topic, of which the majority reported MRI findings, a few radiographic findings and only one ultrasonographic findings. It has proved challenging to extract information from the available scientific literature. Studies are not easily comparable as they use different designs and terminologies (the same diagnostic term is often defined differently from author to author).

Study design is essential in ensuring adequate quality of the obtained results. In six of the retrieved studies, ${ }^{14} 16$ 18-20 22 study participants had not been recruited prospectively, and the assessment of imaging data was therefore retrospective. Study group populations were often heterogeneous in terms of sport types $^{11} 13^{14}{ }^{17-26}$ and gender. ${ }^{16-23}$ Sample sizes of case and control groups were generally small, and case and control groups were sometimes ill matched in terms of age, ${ }^{16} 17192025$ gender ${ }^{16} 1719-2125$ and physical activity. ${ }^{16} 1719202526$ Clinical information (which would convey crucial information concerning possible aetiological factors) was often lacking or incomplete. Moreover, it was difficult to assess whether clinical symptoms were comparable within a study group population, as symptoms were often described solely as 'groin pain'.

\section{Diagnostic confusion}

Overall, definitions and terms used to describe diagnoses in athletes with symphyseal and adductor-related groin pain are not standardised, so that definitions often overlap. The main diagnostic expressions used are 'osteitis pubis' and 'athletic pubalgia' (tables 1-2), but the terminology remains unclear. 'Osteitis pubis' is often used as a diagnostic term, although there is no consensus as to its definition: a clinical entity, a radiological condition or both. Fricker et $a l^{22}$ describe it as a self-limiting disease of the pubic symphysis, marked by erosion of the joint margins followed by healing. Schilders et $a l^{1124}$ refer to it as tenderness on palpation of the pubic symphysis and the presence of inflammatory changes at the symphysis on MRI scans. 
Table 3 Radiographic and ultrasonographic studies

\begin{tabular}{|c|c|c|c|c|c|}
\hline Study & Harris and Murray (1974) & Fricker et al(1991) & Major and Helms (1997) & Besjakov et al(2003) & Kälebo et al(1992) \\
\hline $\begin{array}{l}\text { Case-study group } \\
\text { participants (incl. sports } \\
\text { type, gender, age) }\end{array}$ & $\begin{array}{l}\text { Group 1: } 26 \text { athletes (soccer) } \\
\text { Group 2: } 11 \text { athletes (9 soccer, } \\
1 \text { jockey, } 1 \text { runner) }\end{array}$ & $\begin{array}{l}59 \text { patients ( } 28 \text { running, } 8 \text { soccer, } 8 \text { ice hockey \& others) } \\
50 \text { male, } 9 \text { female. Age } 13-61 \\
44 \text { male \& } 4 \text { female performing sports }\end{array}$ & $\begin{array}{l}11 \text { athletes ( } 7 \text { runners, } \\
1 \text { soccer, } 3 \text { basketball) } \\
9 \text { male, } 2 \text { female. } \\
\text { Age } 20-60\end{array}$ & $\begin{array}{l}20 \text { athletes (17 soccer, } 2 \text { handball, } 1 \text { ice hockey) } \\
\text { Male. Age } 19-35\end{array}$ & $\begin{array}{l}36 \text { patients } \\
28 \text { male, } 8 \text { female. Age } 14-57\end{array}$ \\
\hline Controls & $\begin{array}{l}156 \text { controls of whom some } \\
\text { athletic (number unknown) } \\
\text { Male, Age 17-18 }\end{array}$ & None & $\begin{array}{l}20 \text { controls } \\
11 \text { male, } 9 \text { female. } \\
\text { Age } 18-72\end{array}$ & $\begin{array}{l}\text { Group 1: } 20 \text { men } \\
\text { Group 2: } 120 \text { adults ( } 66 \text { males, } 54 \text { females) Age } \\
\text { 15-90 }\end{array}$ & $\begin{array}{l}\text { Same patients : Ultrasonography of the } \\
\text { contralateral asymptomatic side }\end{array}$ \\
\hline Inclusion criteria & $\begin{array}{l}\text { Group 1: athletes from same } \\
\text { professional soccer team (1 with } \\
\text { groin pain) } \\
\text { Group 2: athletes with history of } \\
\text { groin/lower abdominal pain }\end{array}$ & $\begin{array}{l}\text { Patients with osteitis pubis (clinical signs and positive } \\
\text { radiographic or isotope bone scan findings) }\end{array}$ & $\begin{array}{l}\text { Cases: groin and/or lower } \\
\text { back pain (sciatica) } \\
\text { Controls: Asymptomatic }\end{array}$ & $\begin{array}{l}\text { Cases: Uni or bilat groin pain for }>3 \text { months } \\
\text { Control 1: age-matched men without symptoms, } \\
\text { level of physical activity unknown } \\
\text { Control 2: Not described }\end{array}$ & $\begin{array}{l}\text { Pain and weakness in groin area during } \\
\text { physical activity }\end{array}$ \\
\hline Exclusion criteria & Not described & Not described & Not described & $\begin{array}{l}\text { Control 1: History of sports injury, urologic } \\
\text { complaint, or other pelvic condition }\end{array}$ & Not described \\
\hline $\begin{array}{l}\text { Clinical findings } \\
\text { (1) type of examination } \\
\text { (2) findings }\end{array}$ & None & $\begin{array}{l}\text { (1) Not described } \\
\text { (2) } 70 \% \text { have tenderness at pubic symphysis, } 42 \% \\
\text { adductor-related pain, }\end{array}$ & $\begin{array}{l}\text { (1) Not described } \\
\text { (2) All cases have } \\
\text { tenderness on palpation of } \\
\text { pubic symphysis }\end{array}$ & None & $\begin{array}{l}\text { (1) Not reproducible but details given } \\
\text { (2) All cases have unilateral pain ( } 9 \\
\text { adductor-related, } 13 \text { hamstrings, } 5 \text { rectus } \\
\text { femoris, } 4 \text { gluteal muscle, } 2 \text { rectus } \\
\text { abdominis) }\end{array}$ \\
\hline Radiology protocol & $\begin{array}{l}\text { Group 1-2: Plain PA pelvic view } \\
\text { close to the symphysis with pt } \\
\text { standing on each leg (2) } x \text {-rays) } \\
\text { Controls: Pelvic AP supine }\end{array}$ & $\begin{array}{l}\text { Not described } \\
\text { Radiographs (number unknown) }\end{array}$ & $\begin{array}{l}\text { Plain pelvic AP films (not } \\
\text { defined if supine or } \\
\text { standing) }\end{array}$ & $\begin{array}{l}\text { For all patients: Plain pelvic films in supine } \\
\text { position and additional posistions if necessary }\end{array}$ & $\begin{array}{l}7,5 \mathrm{MHz} \text { linear array transducer } \\
\text { Examiner not described }\end{array}$ \\
\hline $\begin{array}{l}\text { Additional radiological } \\
\text { examinations }\end{array}$ & None & Isotope bone scans (number unknown) & $\begin{array}{l}4 \text { cases } C T, 2 \text { cases MRI } \\
\text { and } 3 \text { cases bone scan }\end{array}$ & None & $\begin{array}{l}\text { Plain radiography to exclude avulsion } \\
\text { fractures }\end{array}$ \\
\hline Evaluation of images & $\begin{array}{l}\text { Group 1-2: } \\
\text { (1) Abnormal width of } \\
\text { symphyseal cleft }(>1 \mathrm{~cm}) \text {, } \\
\text { marginal irregularity, reactive } \\
\text { sclerosis } \\
\text { (2) Instability on stress films } \\
\text { (difference in height of superior } \\
\text { pubic rami }>2 \mathrm{~mm} \text { ) } \\
\text { (3)accentuation of origins of } \\
\text { gracilis muscle } \\
\text { Controls: instability not assessed } \\
\text { due to lack og stress films }\end{array}$ & $\begin{array}{l}1 \text { Radiologist blinded to clinical symptoms } \\
\text { (1) Radiographs: symphyseal changes. Uni/bilateral. Pubic } \\
\text { instability on stress films } \\
\text { (2) Isotope bone scans: normal, mild, moderate or marked } \\
\text { increased isotope uptake. Uni/bilateral. } \\
\text { Presence of obliteration of the symphyseal cleft. }\end{array}$ & Not described & $\begin{array}{l}2 \text { Radiologists } \\
\text { Changes divided into } 4 \text { groups according to } \\
\text { grading scale (no bone changes/slight/ } \\
\text { intermediate/advanced changes) classifying visible } \\
\text { changes in the pubic bones and symphysis }\end{array}$ & $\begin{array}{l}\text { Proximal tendons and tendomuscular } \\
\text { junctions of recus femoris, rectus abdominis, } \\
\text { adductor muscles, hamstring muscles and } \\
\text { gluteal muscles } \\
\text { Tendons scanned longitudinally and } \\
\text { transversely to identify lesions }\end{array}$ \\
\hline $\begin{array}{l}\text { Reproducibility of } \\
\text { radiology }\end{array}$ & None & None & None & Interobserver agreement & None \\
\hline 'Gold standard" used & None & None & None & None & Surgery in 10 patients \\
\hline Conclusions/results & $\begin{array}{l}\text { Group 1: abnormalities in } 76 \% \\
\text { Group 2: abnormalities in } 81 \% \\
\text { Controls: abnormalities in } 45 \%\end{array}$ & $\begin{array}{l}\text { Males: } 38 \% \text { with unilateral and } 63 \% \text { with bilateral pain } \\
\text { show corresponding positive isotope bone scans. } 38 \% \text { with } \\
\text { unilateral and } 43 \% \text { with bilateral pain show corresponding } \\
\text { positive radiographic changes. } \\
\text { Women with unilateral pain show no matching and women } \\
\text { with bilateral pain show } 100 \% \text { matching with radiological } \\
\text { findings }\end{array}$ & $\begin{array}{l}\text { Cases: All cases have } \\
\text { erosions, areas of sclerosis } \\
\text { or offset at pubic } \\
\text { symphysis. } \\
4 \text { cases: Avulsion of inferior } \\
\text { part of symphysis } \\
\text { Controls: } 6 \text { ptts over age } 55 \\
\text { have mild sclerosis at pubic } \\
\text { symphysis }\end{array}$ & $\begin{array}{l}\text { Cases: } 9 \text { slight, } 9 \text { intermediate, } 2 \text { advanced } \\
\text { changes } \\
\text { Control 1: } 3 \text { none,17 slight changes } \\
\text { Control 2: } 40(42) \text { none, } 65 \text { (64) slight, 15(14) } \\
\text { intermediate } \\
\text { Increase of abnormalities with age in control } \\
\text { group 2 }\end{array}$ & $\begin{array}{l}28 \text { of } 36 \text { cases have tendon lesions at the } \\
\text { corresponding painful areas. } \\
\text { Location: } 12 \text { in proximal tendon, } 11 \text { in } \\
\text { tendomuscular junction and } 5 \text { in } \\
\text { tendon-bone junction } \\
9 \text { of } 10 \text { surgically treated cases: findings } \\
\text { similar to ultrasound }\end{array}$ \\
\hline
\end{tabular}




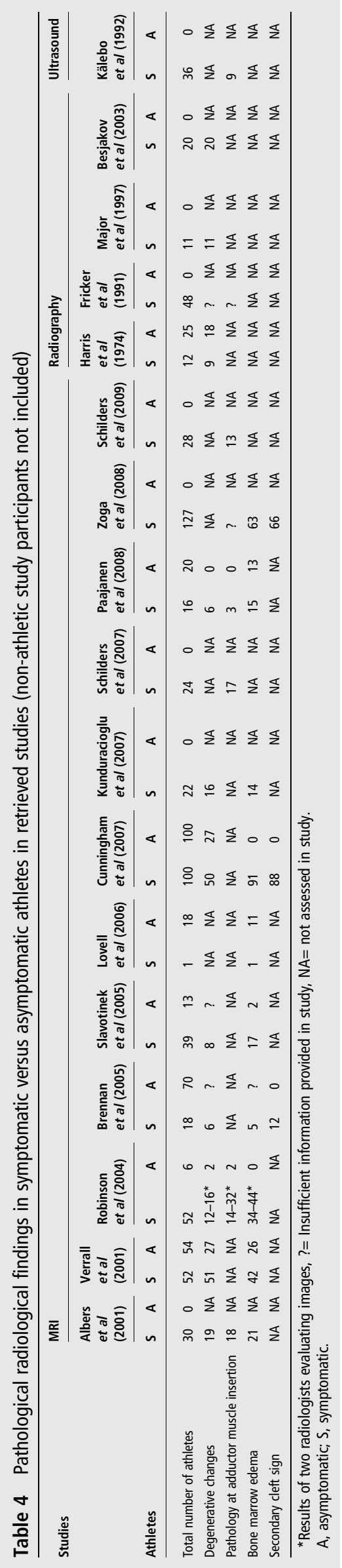

Cunningham et $a l^{17}$ define it radiologically as the presence of para-articular bone marrow oedema (BMO), remote from the adductor muscle attachment, Brennan et al ${ }^{14}$ as symphyseal degenerative changes on conventional radiographs and MRI, and as BMO in the medial pubic bones. Zoga et al ${ }^{20}$ define it on MRI as BMO spanning the symphysis. For others, ${ }^{10} 19$ 'osteitis pubis' represents a combination of pubic groin pain, local tenderness on palpation of the symphysis, painfulresisted bilateral hip adduction and the presence of pubic BMO on MRI. 'Athletic pubalgia' is a quite unspecific term that describes sports-related pain at the site of the pubic symphysis, and appears in two of the retrieved MRI studies: ${ }^{18} 20$ it is used as a diagnostic term when the physical examination of athletes is indeterminate, and the cause of groin pain is unidentifiable. ${ }^{18}$

Pubic instability is another ill-defined radiological and clinical term. Standard radiography of the pelvis consists of static inlet and outlet anteroposterior views, but evaluating pathological vertical shift motion between the symphyseal joint surfaces requires a dynamic stress examination of the pelvis. This was first achieved by Chamberlain ${ }^{28}$ in 1930 using single-leg-stance (flamingo) views. Despite the methodological shortcomings and a lack of demographic information concerning the study population, normal motion at the pubic symphysis was measured up to $2 \mathrm{~mm}$ in this study and others. ${ }^{29} 30$ One recent study, however, reported a physiological joint motion of up to $5 \mathrm{~mm} .{ }^{31}$ There is a need for further imaging studies defining and assessing pelvic instability in athletes, and correlating dynamic radiographic findings with clinical symptoms, before pubic instability can be considered a diagnosis.

\section{Reliability}

Few studies assess the reliability and reproducibility of radiological findings in symphyseal and adductor-related groin pain. An accurate interpretation of images depends on functional and reliable imaging parameters that allow multiple observers to reproduce the same results time after time and thereby establish a correct diagnosis. Even though numerous radiological parameters are described in the literature, it remains unclear how subjective their interpretation actually is, and further reliability assessments would therefore improve current practice.

\section{Gold standards}

In a radiological setting, a gold standard is the most accurate diagnostic test against which other radiological modalities are evaluated and compared. Ideally, an MRI scan should be held up against another test known to be the best available under reasonable conditions, to evaluate if the interpretation of MRI is correct and in accordance with the gold standard.

Seven MRI studies and the US study attempted to use a 'gold standard', ${ }^{11} 1^{14}$ 17-20 2324 whereas the radiographic studies used none. In two MRI studies, ${ }^{14}{ }^{17}$ fluoroscopy-guided contrast injection into the pubic symphyseal cleft in a group of symptomatic athletes was used as a gold standard with which to compare MRI scans. The aim was to demonstrate the presence of a primary and/or secondary cleft sign (defined as extension of contrast material either lateral to the midline or inferior to the symphyseal joint ${ }^{14}$ ) and assess whether it was visible on both imaging modalities. However, at present, there exists no evidence to prove the exact nature of a secondary cleft and what significance it has for long-standing pain. In two other MRI studies, ${ }^{11} 24$ an injection of a local anaesthetic and steroid into the pubic cleft was performed under US guidance in a group of symptomatic athletes in an attempt to alleviate 
pain symptoms. In these studies, symptomatic athletes were MRI scanned with intravenous gadolinium, and the authors interpreted the presence of contrast enhancement at the adductor enthesis as enthesitis. However, both studies reported immediate alleviation of pain symptoms upon pubic cleft injection in all participants, whereas only 17 of 24 individuals displayed contrast enhancement at the adductor muscle insertion in the first study, ${ }^{11}$ and 13 of 28 individuals in the second study. $^{24}$

Three MRI studies used surgery as a reference to evaluate radiological results. ${ }^{18-20}$ Zoga et al ${ }^{20}$ compared MRI findings retrospectively with physical examination findings and surgical results in a group of 141 patients, of whom 102 had undergone surgery. They reported MRI sensitivities and specificities of $68 \%$ and $100 \%$ compared with surgery for rectus abdominis tendinous injuries, and of $86 \%$ and $89 \%$, respectively, for adductor tendon injuries. However, MRI scans were not easily comparable as they differed in terms of scanning protocols, field strengths and anatomical areas covered. Paajanen et $a l^{19}$ performed non-randomised surgery on 8 of 18 athletes with osteitis pubis who did not respond to conservative therapy. They found that although operated athletes had more symptoms and a longer pain history than patients treated conservatively, they recovered faster than the non-operated patients. Moreover, they reported no statistical difference in the degree of BMO between the groups of operated and non-operated athletes.

In the US study, ${ }^{23} 10$ of 36 patients were treated surgically, and in 9 of these patients, the surgical findings correlated well with the ultrasonographic findings (of which four patients had ruptured adductor tendons). However, as the nature and aetiology of pathological findings in athletes with groin pain are largely unknown, these gold standards cannot be considered to be better tests than MRI scans.

Owing to the methodological shortcomings listed above, we have attempted to classify the results of the retrieved studies according to the actual radiological findings reported by the authors, and not according to diagnostic terms such as 'osteitis pubis', 'athletic pubalgia' or 'adductor dysfunction', as these diagnoses differ between studies. Radiological findings associated with symphyseal and adductor-related groin pain can therefore be classified into four groups: (1) degenerative changes at and around the symphyseal joint, (2) pathological changes at the adductor muscle insertion to the pubic bone, (3) pubic BMO and (4) the presence of a secondary cleft sign (table 4).

\section{Degenerative changes around the symphyseal joint}

The symphyseal joint is a fibrocartilaginous joint with a central disc interposed between two hyaline cartilage-covered joint surfaces. Normally, these joint surfaces are smooth and well delineated, the central disc is contained within the joint capsule and there is often a small physiological fluid-filled space inside the disc (called a primary cleft). ${ }^{32}$ Degenerative changes in and around this joint consist of joint surface erosions and irregularities, subchondral sclerosis and cysts, joint space widening or narrowing, central disc herniation and bony proliferation (beaking) at the superior margins of the joint. All radiographic studies $^{21} 222526$ and seven MRI studies ${ }^{12-18}$ assessed degenerative changes at the symphyseal joint. Their prevalence is variable among studies and depends on whether the study participants are symptomatic or not. In two radiographic studies, almost all symptomatic athletes presented chronic degenerative changes at the pubic symphysis of greater severity and prevalence than the corresponding non-athletic control groups. ${ }^{21} 26$ Moreover, radiographic studies showed symphyseal joint changes in around $70-80 \%$ of asymptomatic athletes ${ }^{21} 25$ but much fewer $(45-65 \%)$ in non-athletic controls, ${ }^{25}{ }^{26}$ whereas the prevalence of degenerative changes increased with age. ${ }^{26}$

In MRI studies, the prevalence of degenerative symphyseal changes varied from $20 \%{ }^{16}$ to $33 \%,{ }^{14} 37 \%,{ }^{19} 50 \%,{ }^{17} 63 \%,{ }^{18}$ $73 \%{ }^{13}$ and $98 \%{ }^{12}$ of symptomatic athletes, whereas the prevalence recorded for asymptomatic athletes varied from $0 \%{ }^{19}$ to $27 \%,{ }^{17} 33 \%{ }^{16}$ and $50 \% .^{12}$ Thus, even though degenerative changes at the symphyseal joint can be observed in both symptomatic and asymptomatic individuals, there is some indication that they are more commonly found in athletes with longstanding symphyseal pain than in asymptomatic athletes.

\section{Pathology at the adductor muscle insertions}

Adductor-related groin pain is a diagnostic entity that describes pain related to the adductor muscle insertions at the pubic bones. ${ }^{33}$ Schilders et al ${ }^{1124}$ considered adductor-related pain to be present if the clinical examination demonstrated tenderness at the adductor enthesis, and pain on passive adductor stretching and resisted adduction of the thigh. In another study, ${ }^{16}$ the authors used the term 'adductor-related' as well as the term 'adductor dysfunction' for clinical adductor tenderness and pain exacerbated on resisted adduction, which is identical to the diagnostic entity adductor-related groin pain. ${ }^{33}$ There exists at present no radiological grading scale to evaluate the severity of pathology at the adductor enthesis site.

Six of the retrieved MRI studies, ${ }^{11} 16 \quad 18-2024$ one radiographic $^{22}$ and the US study ${ }^{23}$ reported findings on groin pain originating from the adductor muscle insertions. The prevalence of adductor enthesis pathology at MRI (defined in three studies as contrast enhancement at the site of the adductor enthesis ${ }^{11} 16^{24}$ ) was variable: $71 \%{ }^{11}$ in a group of symptomatic professional athletes, and $46 \%{ }^{24}$ in a group of recreational athletes with adductor-related groin pain. In another study, ${ }^{19}$ three of three athletes with positive clinical adductor-type pain showed increased signal intensity at the site of the adductor muscle attachment.

\section{Pubic BMO}

$\mathrm{BMO}$ is visible on fluid-sensitive MRI sequences as increased signal intensity within the pubic bone marrow. It has been the subject of considerable interest in several studies, as its presence is suspected of being correlated to the severity of long-standing pubic pain. ${ }^{12}$ Even though BMO is a commonly evaluated radiological finding, its assessment is not standardised. There exists no reliable and reproducible grading scale. Instead, BMO was graded subjectively according to a Likert scale $(0=$ no changes, $1=$ mild, $2=$ moderate, $3=$ severe $)$ in five studies, ${ }^{10} 12151619$ and was moreover graded according to its regional extent at the pubic symphysis (less or more than $2 \mathrm{~cm}$ ) in two studies. ${ }^{12}{ }^{15}$ One of these studies ${ }^{15}$ evaluated the interobserver variation for the grading of BMO, yielding a $\kappa$ value of 0.85 . In four other articles, the presence of $\mathrm{BMO}$ was recorded but not graded. ${ }^{1314} 171820$ Thus, the assessment of the severity of $\mathrm{BMO}$ is rather subjective.

$\mathrm{BMO}$ is often found in symptomatic athletes: studies have reported its prevalence as varying from $28 \%{ }^{14}$ to $44 \%,{ }^{15}$ $50 \%,{ }^{20} 64 \%,{ }^{13} 70 \%,{ }^{18} 81 \%,{ }^{12} 91 \%,{ }^{17} 94 \%{ }^{19}$ and $100 \% .^{10}$ However, it is often present in asymptomatic athletes as well: the prevalence in our retrieved studies spanned widely from $0 \%{ }^{17}$ to $15 \%,{ }^{15} 48 \%,{ }^{12} 61 \%{ }^{10}$ and $65 \%$. $^{19}$ 
Two additional MRI studies examining exclusively asymptomatic athletes reported the prevalence of pubic BMO among their study participants to be $23 \%{ }^{34}$ and $57 \%,{ }^{35}$ respectively. Interestingly, in the latter study, ${ }^{35}$ the prevalence of pubic BMO was $50 \%$ in a group of sedentary matched asymptomatic controls. Overall, pubic BMO seems to be more prevalent and more severe in symptomatic versus asymptomatic athletes.

\section{Secondary cleft sign}

The secondary cleft sign is mentioned in three of the retrieved MRI studies. ${ }^{14} 1720$ It has been defined by Brennan et al ${ }^{14}$ as any evidence at the symphyseal cleft injection of extension of contrast material either lateral to the midline or inferior to the joint, by Cunningham et $a l^{17}$ as an abnormal inferior extension of the cleft in symphyseal fibrocartilage, and by Zoga et $a l^{20}$ as a curvilinear area with the signal intensity of fluid extending inferolaterally from the inferior aspect of the symphysis on coronal images. Its prevalence was $52 \%,{ }^{20} 67 \%{ }^{14}$ and $88 \%,{ }^{17}$ respectively, among athletes with symphyseal groin pain in these studies, and it corresponded to the side of symptoms in all cases. Asymptomatic controls in these studies presented no secondary cleft sign, irrespective of whether they were athletes $^{14}{ }^{17}$ or sedentary. ${ }^{20}$

Authors interpret the secondary cleft sign as a possible consequence of a microtear or traction force at the site of the adductor attachment to the pubic bone, and thus as an indirect sign of a lesion at the adductor muscle attachment site. However, its significance is still debatable.

\section{CONCLUSION}

Radiological evaluation of long-standing symphyseal and adductor-related groin pain remains a challenging task. Current evidence is based on relatively few heterogeneous studies of varying methodological quality. Four main radiological findings seem to appear consistently: degenerative changes at the pubic symphyseal joint, pathology at the adductor muscle insertions at the pubic bones, pubic BMO and the secondary cleft sign. The existing diagnostic terminology is confusing, and the interpretation of radiological pathological changes would benefit from imaging studies using a more systematic approach. The methodological quality of such studies would be improved by including homogeneous study groups (in terms of age, sex and sport types), well-matched control groups, reproducible clinical examinations and identical, well-designed radiological protocols.

\section{What does this paper add?}

- Radiological evaluation of long-standing symphyseal and adductor-related groin pain is based on relatively few heterogeneous studies of varying methodological quality.

- The existing diagnostic terminology is confusing.

- Four main radiological findings appear: degenerative changes at the pubic symphyseal joint, pathology at the adductor muscle insertions at the pubic bones, pubic bone marrow oedema and the secondary cleft sign.

- This topic requires further systematic research.

Correction notice This article has been corrected since it was published Online First. An author affiliation for Per Hölmich was missing, which has now been added.
Contributors SB has substantially contributed to the conception, design, drafting and revision of the article, as well as the analysis and interpretation of data. KT, $\mathrm{MBJ}$ and $\mathrm{PH}$ have substantially contributed to the conception, design and revision of the article, as well as the interpretation of data and final approval of the version to be published.

\section{Competing interests None.}

Provenance and peer review Not commissioned; externally peer reviewed.

\section{REFERENCES}

1 Orchard J, Seward H. Epidemiology of injuries in the Australian Football League, seasons 1997;2000. Br J Sports Med 2002;36:39-44.

2 Fricker PA. Management of groin pain in athletes. Br J Sports Med 1997;31:97-101.

3 Lynch SA, Renstrom PA. Groin injuries in sport: treatment strategies. Sports Med 1999;28:137-44.

4 Renstrom P, Peterson L. Groin injuries in athletes. Br J Sports Med 1980;14:30-6.

5 Ekberg 0, Persson NH, Abrahamsson PA, et al. Longstanding groin pain in athletes. A multidisciplinary approach. Sports Med 1988;6:56-61.

6 Mullens FE, Zoga AC, Morrison WB, et al. Review of MRI technique and imaging findings in athletic pubalgia and the "sports hernia". Eur J Radiol 2012;81:3780-92.

7 Tavernier T, Cotten A. High- versus low-field MR imaging. Radiol Clin North Am 2005;43:673-81.

8 Siegel J, Templeman DC, Tornetta P III. Single-leg-stance radiographs in the diagnosis of pelvic instability. J Bone Joint Surg Am 2008;90:2119-25.

9 Alicioglu B, Kartal O, Gurbuz $\mathrm{H}$, et al. Symphysis pubis distance in adults: a retrospective computed tomography study. Surg Radiol Anat 2008;30:153-7.

10 Lovell G, Galloway H, Hopkins W, et al. Osteitis pubis and assessment of bone marrow edema at the pubic symphysis with MRI in an elite junior male soccer squad. Clin J Sport Med 2006;16:117-22.

11 Schilders E, Bismil Q, Robinson P, et al. Adductor-related groin pain in competitive athletes. Role of adductor enthesis, magnetic resonance imaging, and entheseal pubic cleft injections. J Bone Joint Surg Am 2007;89:2173-8.

12 Verrall GM, Slavotinek JP, Fon GT. Incidence of pubic bone marrow oedema in Australian rules football players: relation to groin pain. $\mathrm{Br}$ J Sports Med 2001;35:28-33.

13 Kunduracioglu B, Yilmaz C, Yorubulut $M$, et al. Magnetic resonance findings of osteitis pubis. J Magn Reson Imaging 2007;25:535-9.

14 Brennan D, O'Connell MJ, Ryan M, et al. Secondary cleft sign as a marker of injury in athletes with groin pain: MR image appearance and interpretation. Radiology 2005:235:162-7.

15 Slavotinek JP, Verrall GM, Fon GT, et al. Groin pain in footballers: the association between preseason clinical and pubic bone magnetic resonance imaging findings and athlete outcome. Am J Sports Med 2005;33:894-9.

16 Robinson P, Barron DA, Parsons W, et al. Adductor-related groin pain in athletes: correlation of MR imaging with clinical findings. Skeletal Radiol 2004;33:451-7.

17 Cunningham PM, Brennan D, O'Connell M, et al. Patterns of bone and soft-tissue injury at the symphysis pubis in soccer players: observations at MRI. AJR Am J Roentgenol 2007;188:W291-6.

18 Albers SL, Spritzer CE, Garrett WE, et al. MR findings in athletes with pubalgia. Skeletal Radiol 2001;30:270-7.

19 Paajanen $\mathrm{H}$, Hermunen $\mathrm{H}$, Karonen J. Pubic magnetic resonance imaging findings in surgically and conservatively treated athletes with osteitis pubis compared to asymptomatic athletes during heavy training. Am J Sports Med 2008;36:117-21.

20 Zoga AC, Kavanagh EC, Omar IM, et al. Athletic pubalgia and the "sports hernia": MR imaging findings. Radiology 2008;247:797-807.

21 Major NM, Helms CA. Pelvic stress injuries: the relationship between osteitis pubis (symphysis pubis stress injury) and sacroiliac abnormalities in athletes. Skeletal Radiol 1997;26:711-17.

22 Fricker PA, Taunton JE, Ammann W. Osteitis pubis in athletes. Infection, inflammation or injury? Sports Med 1991;12:266-79.

23 Kalebo P, Karlsson J, Sward L, et al. Ultrasonography of chronic tendon injuries in the groin. Am J Sports Med 1992;20:634-9.

24 Schilders E, Talbot JC, Robinson P, et al. Adductor-related groin pain in recreational athletes: role of the adductor enthesis, magnetic resonance imaging, and entheseal pubic cleft injections. J Bone Joint Surg Am 2009;91:2455-60.

25 Harris NH, Murray RO. Lesions of the symphysis in athletes. Br Med J 1974;26:211-14.

26 Besjakov J, Von Scheele C, Ekberg O, et al. Grading scale of radiographic findings in the pubic bone and symphysis in athletes. Acta Radiol 2003;44:79-83.

27 Holmich P, Holmich LR, Bjerg AM. Clinical examination of athletes with groin pain: an intraobserver and interobserver reliability study. Br J Sports Med 2004;38:446-51.

28 Chamberlain WE. The symphysis pubis in the roentgen examinationof the sacroiliac joint. Am J Roentgenol Radium Ther 1930;24:621-5.

29 Walheim G, Olerud S, Ribbe T. Mobility of the pubic symphysis. Measurements by an electromechanical method. Acta Orthop Scand 1984;55:203-8.

30 Walheim GG, Olerud S, Ribbe T. Motion of the pubic symphysis in pelvic instability. Scand J Rehabil Med 1984;16:163-9. 


\section{Review}

31 Garras DN, Carothers JT, Olson SA. Single-leg-stance (flamingo) radiographs to assess pelvic instability: how much motion is normal? J Bone Joint Surg Am 2008;90:2114-18.

32 Putschar WG. The structure of the human symphysis pubis with special consideration of parturition and its sequelae. Am J Phys Anthropol 1976;45:589-94.

33 Holmich P. Long-standing groin pain in sportspeople falls into three primary patterns, a "clinical entity" approach: a prospective study of 207 patients. $\mathrm{Br}$ J Sports Med 2007;41:247-52.
34 Silvis ML, Mosher TJ, Smetana BS, et al. High prevalence of pelvic and hip magnetic resonance imaging findings in asymptomatic collegiate and professional hockey players. Am J Sports Med 2011:39:715-21.

35 Paajanen $\mathrm{H}$, Hermunen $\mathrm{H}$, Karonen J. Effect of heavy training in contact sports on MRI findings in the pubic region of asymptomatic competitive athletes compared with non-athlete controls. Skeletal Radiol 2011:40:89-94.

$\underline{\underline{w}}$

$c$

O)

$\frac{\text { 을 }}{\text { n }}$

\

$\overrightarrow{\vec{二}}$

음 